\title{
Early development in infants at risk of childhood apraxia of speech: A longitudinal investigation.
}

Chantelle Highman ${ }^{1,2}$, Neville W. Hennessey ${ }^{1 *}$, Suze Leitão ${ }^{1}$, \& Jan P. Piek ${ }^{1}$

${ }^{1}$ School of Psychology and Speech Pathology, Curtin Health Innovation Research Institute, Curtin University

${ }^{2}$ Andrea Way Child Development Centre

Chantelle Highman, School of Psychology and Speech Pathology, Curtin University, Kent St, Bentley, Perth, Western Australia, 6102. Email: C.Highman@curtin.edu.au

Neville Hennessey, School of Psychology and Speech Pathology, Curtin University, Kent St, Bentley, Perth, Western Australia, 6102. Email: N.Hennessey@curtin.edu.au Tel: +61 892662553

Suze Leitão, School of Psychology and Speech Pathology, Curtin University, Kent St,

Bentley, Perth, Western Australia, 6102. Email: $\underline{\text { S.Leitao@curtin.edu.au }}$

Tel: +6189266 7620

Jan Piek, School of Psychology and Speech Pathology, Curtin University, Kent St, Bentley, Perth, Western Australia, 6102. Email: J.Piek@curtin.edu.au

Corresponding Author: Neville

Keywords: Infancy, Speech Sound Disorders, Childhood Apraxia of Speech, Development, Speech Motor 


\begin{abstract}
This study examined early features of the heritable phenotype associated with childhood apraxia-of-speech (CAS). We compared speech and language development from 9 to 24 months of age in eight children at familial risk of CAS to that of eight infants with no such family history. At-risk infants scored lower on expressive language, speech development, and fine motor skills. Results support a broad, heritable verbal trait deficit for children at risk of CAS. Single case analyses showed poor prelinguistic speech development can dissociate from emerging receptive language and conceptualisation skills, consistent with a deficit originating in speech motor control.
\end{abstract}


Childhood apraxia of speech (CAS) is a severe speech sound disorder with a low prevalence (e.g., .1 to .2\%, Shriberg, Aram \& Kwiatkowski, 1997). Behavioural symptoms of CAS include a high incidence of consonant and vowel errors, inconsistency in those errors, difficulty in sequencing speech units, and prosodic difficulties (Davis, Jakielski, \& Marquardt, 1998). CAS has been viewed as a speech motor planning or programming disorder (Shriberg et al., 1997; Terband, Maassen, Guenther, \& Brumberg, 2009), however, children with CAS commonly show language impairment and overlap in features with other developmental speech (e.g., phonological) and language disorders leading some researchers to question the difference in their aetiology (Marion, Sussman, \& Marquardt, 1993).

Studies have also shown a variety of speech and language disorders aggregate in firstdegree family relatives of children diagnosed with CAS (Lewis et al., 2004; Lewis et al., 2006). A similar pattern of family aggregation has been observed for speech-disordered children without CAS (Lewis et al., 2004). These findings are consistent with the hypothesis of a broad heritable verbal trait deficit underlying developmental speech and language disorders (Lewis et al., 2004) where children with CAS sit at the more severe end of a continuum involving, but not exclusively, speech motor difficulties (cf. Lewis et al., 2006). From this perspective children with CAS share in a range of higher-level (e.g., linguistic) deficits used to explain phonological and language disorders in children (Bishop, 2006; Lewis et al., 2006).

Consistent with dynamic systems theory, a number of researchers emphasise the role of interaction during development between cognitive systems and external factors on the emergence of complex behaviours including language (Evans, 2002; Mitchell, 1995; Piek, 2006). Furthermore, a deficit in one domain can, over time, constrain development in other domains (Bishop, 1997, 2006). From a developmental perspective, therefore, CAS may arise 
from a core deficit in speech motor control that constrains the emergence of associated phonological, lexical and other higher-order linguistic processes (Maassen, 2002), thereby explaining the overlap in symptomatology between CAS and other types of speech and language disorders.

Longitudinal investigations beginning from infancy provide an opportunity to disentangle core deficits from subsequent deficits and provide some insight into aetiology for developmental disorders such as CAS (Bishop, 1997; Maassen, 2002). Do deficits leading to diagnosis of CAS in childhood begin in the speech motor system in infancy, or do they have a broader locus as might be proposed by the verbal trait deficit hypothesis? However, studies examining early development in children at-risk, or later showing features, of CAS have mostly used retrospective reports from parents (e.g., Highman, Hennessey, Sherwood, \& Leitão, 2008). While these studies show limited and late development of prelinguistic babbling consistent with a speech motor origin to the disorder (Davis \& Velleman, 2000; Maassen, 2002), children with expressive language disorders can also show delays in early babbling (Highman et al., 2008; Oller, Eilers, Neal, \& Schwartz, 1999). Babbling may be delayed or atypical for other reasons than speech motor difficulties.

\section{The Present Study}

In the present study eight infants at 9 months of age with a family history of suspected CAS were recruited for participation in a longitudinal investigation until 2 years. All of the atrisk children were biological siblings of children with a clinical diagnosis and history of treatment for CAS. These at-risk siblings were compared in terms of their development to eight age-matched typically developing (TD) infants. Overall group patterns of performance can be informative about a broader phenotype consistent with a verbal trait deficit (Lewis et al., 2004), even when some siblings do not go on to receive a diagnosis or the study timeframe does not allow diagnosis to be confirmed (Koster et al., 2005). The first aim of the 
present study was, therefore, to examine the early presentation and developmental trajectory of infants at familial risk of suspected CAS, and in doing so, profile the heritable phenotype associated with suspected CAS at this early stage of development. We included assessments of speech sound development, expressive and receptive language, social communication skills, as well as measures of gross and fine motor development to test the hypothesis that early deficits in siblings at risk of CAS would encompass domains beyond the speech motor system suggestive of a heritable verbal trait deficit.

Our second aim was to examine infants with poor expressive language outcomes at 2 years, who are deemed to be at increased risk of ongoing speech and language problems, for early features of CAS (cf. Davis \& Velleman, 2000). Consistent with the developmental account, infants at increased risk of CAS may show differences in early development that are confined to measures of speech sound development and expressive language. Following Levelt, Roelofs and Meyer (1999, see, also Maassen, 2002), receptive language skills and conceptual knowledge were assumed to develop independently of the speech motor system prior to the onset of intentional speech at around 12 months of age. We used statistical tests for a dissociation between measures that are appropriate for single cases (e.g., Crawford, Garthwaite \& Grey, 2003) to determine whether deficits in prelinguistic speech motor development (e.g., at 9 months) could be found in the presence of intact conceptual and receptive language development consistent with a speech motor origin to the disorder (Davis \& Velleman, 2000).

\section{Method}

\section{Participants}

Sixteen infants and their primary caregivers (all mothers) took part in the study. All infants were from monolingual English home environments with no identified medical, cognitive or physical disability, based on parent report. Socio-economic status, estimated by 
postcode data, was predominantly middle-class. All at-risk infants were referred for an audiological assessment prior to participation in the study to confirm normal peripheral hearing acuity and middle ear function.

The at-risk group consisted of eight biologically related siblings (four boys and four girls) of children with a clinical diagnosis of CAS made by a qualified speech-language pathologist. The families participating in the study were recruited through speech-language pathologists in the ... metropolitan area. No specific guidance on which particular features were diagnostic of CAS was provided. Although the children diagnosed with CAS were not directly assessed as part of this study, all were receiving or had received treatment for their apraxia of speech symptoms and no other co-morbidity, such as autism, was identified. In response to the question "is there a family history of speech, language or literacy problems?" all parents identified an older sibling (7 older male siblings, 1 not specified) as having been diagnosed with CAS. Two of those parents also identified a third sibling as having "speech therapy" although the diagnosis was not specified. A third parent also identified 3 cousins of their children with a CAS diagnosis. Data were first collected on the infants when they were 9 months of age with two exceptions: at-risk participants 3 (P3) and 5 (P5) were 10 months and 12 months, respectively, when the study commenced.

The TD group consisted of eight age-matched infants (four boys and four girls) with no significant health, medical or developmental issues, who did not have a family history of speech, language or literacy difficulties (based on parent report). Families were recruited with the assistance of child health nurses in the ... metropolitan area, who informed parents of TD infants of the study. Independent samples t-tests confirmed no significant differences in chronological age between the two groups for the $9(t(13)=0.24, p=.82), 12(t(14)=0.37, p$ $=.72), 15(t(14)=0.40, p=.69), 18(t(14)=1.95, p=.07)$, and $24(t(10)=0.61, p=.56)$ months data collection points. 


\section{Materials}

Ages and Stages Questionnaire (ASQ, Bricker et al., 1999). The ASQ parent report questionnaire was used to evaluate development in the areas of communication, gross motor, fine motor, problem solving and personal-social. Psychometric properties of the ASQ are reported to be adequate in terms of internal consistency (Cronbach's alpha .72 to .79), testretest reliability (94\%), inter-observer reliability (percentage agreement 94\%), and concurrent validity was reported to be $72 \%$ and $86 \%$ in terms of sensitivity and specificity, respectively (Boyce \& Poteat, 2005; Bricker et al., 1999).

\section{Communication and Symbolic Behaviour Scales (CSBS) - Caregiver}

Questionnaire (Wetherby \& Prizant, 2002). The CSBS assesses communication abilities across a number of domains in infants and toddlers (Wetherby, Allen, Cleary, Kublin, \& Golstein, 2002; Wetherby \& Prizant, 2002). The Caregiver Questionnaire asks the parent to rate the presence and frequency of occurrence of a range of communication behaviours for their child (e.g., from the sounds cluster: 'Does your child use a variety of different consonant sounds, such as "ba”, "ga", "ta", and "da”, either in vocal play or in words?'). The questionnaire allows standard scores (M 10 SD 3) and percentile ranks to be calculated for seven cluster areas and three composites. The emotion and eye gaze, communication and gestures clusters combine for the social composite; the sounds and words clusters combine for the speech composite; and the understanding and object use clusters combine for the symbolic composite. The CSBS has demonstrated strong or good levels of internal consistency (Cronbach's alpha .95 to .97 ), test-retest stability (.65 to .93 ), and good content, face, construct and criterion validity are also evident (Wetherby et al., 2002; Wetherby \& Prizant, 2002).

\section{Receptive-Expressive Emergent Language Test, third edition (REEL-3, Bzoch,}

League, \& Brown, 2003). The REEL-3 is a clinician-administered test of emerging language 
in children from birth to three years of age. Parents are asked a standard set of questions and raw scores for the receptive and expressive language scales are converted to standard (ability) scores (M 100 SD 15) and percentile ranks. The REEL-3 has sound psychometric properties, with good internal consistency (coefficients of .92 and .93 for receptive and expressive subtests, respectively), and Cohen's kappa (.99) showed strong test-retest reliability and interrater reliability (Hurford \& Stutman, 2004). Content, criterion-related and construct validity were also found to be acceptable (Hurford \& Stutman, 2004).

\section{MacArthur-Bates Communication Development Inventories (CDI, Fenson et al.,}

1993). The Words and Sentences version of the CDI is a parent-completed tool designed to measure expressive vocabulary, morphological and syntactic development in children aged 16 to 30 months. The tool has been utilised extensively in research and has demonstrated excellent psychometric properties (Fenson et al., 1993).

\section{Procedure}

Parents were provided with written and verbal information about the study and gave written consent for their child to participate. Ethics clearance was obtained from the $\ldots$ and ... human research ethics committees.

Data were collected mostly within two weeks of the infants reaching 9, 12, 15, 18 and 24 months. The first author, a qualified speech-language pathologist, carried out all clinicianadministered assessments and scored all assessments following the standardized procedures. Not all measures were included at all assessments. The REEL-3, CSBS caregiver questionnaire and the ASQ were administered at the 9, 12 and 18 months face-to-face assessment sessions. At 15 months the CSBS caregiver questionnaire was posted to the parents and scored on their return, and the REEL-3 was administered over the phone with the primary caregiver. Data for 24 months were collected via the CSBS caregiver questionnaire and CDI with questionnaires sent out and returned in the post. 


\section{Data Analysis Approach}

Only measures showing a significant difference using standard scores (SS) between the at-risk and TD groups using independent groups $t$-tests (or Mann-Whitney $\mathrm{U}$ when normality was violated) from one time point at least were analysed further. Multilevel linear modelling (MLM), which treats time as a nested variable within participants, was used to examine the developmental trajectories in the raw scores of those significant measures. The restricted maximum likelihood estimation procedure appropriate for small sample sizes was used (Heck, Thomas, \& Tabata, 2010) and we tested linear and quadratic trends across time points as fixed effects. The contribution of group and the interaction of group and time were also evaluated as fixed effects. The autoregressive covariance structure was used to account for serial dependency in repeated measurements over time. MLM is able to handle missing data for some time points without excluding participants $(6.25 \%$ of data were missing in the present study).

Single case analysis. The revised standardised difference test (RSDT; Crawford et al., 2003), was used to test for a dissociation between measures of prelinguistic conceptual and receptive language development and speech motor development (i.e., at 9 months of age) for those at-risk participants with delayed expressive language at two years of age. The TD infants were used as the reference group for these single case analyses. The social composite of the CSBS caregiver questionnaire reflects development in the broad area of communication, such as communicative intent and message conceptualisation skills. Example questions include When your child is playing with a toy, does he/she look at you and then back at the toy? (emotion and eye-gaze cluster), Does your child try to get your attention when you are busy doing something, such as when you are talking with an adult or preparing a meal? (communication cluster), and Which of the following gestures have you seen your child use? (gestures cluster). The areas assessed, therefore, include modalities of early 
communicative function that are not reliant on expressive language and articulation development. The CSBS sounds subtest was used as a standardised measure of speech motor development, reflecting the infant's production of syllabic articulatory gestures. The RSDT, therefore, allowed us to test whether infants at risk of CAS can show poor speech sound development but normal conceptual development as measured by the social composite. We also tested for dissociation between the receptive and expressive ability scores of the REEL3 (cf. Highman, Leitão, Hennessey \& Piek, 2012).

\section{Results}

\section{ASQ}

Growth curve analysis of ASQ communication scores revealed a significant linear trend over time, $b=-14.18(95 \% \mathrm{CI}=-27.2$ to -1.2$), t(14.84)=-2.33, p=.034$. The negative coefficient shows communication scores declined in a linear fashion with age (see Figure 1). Group, when added to the model, was not statistically significant, $p=.077$, and the interaction between group and time was not significant, $p=.398$. Four at-risk infants scored below the ASQ communication cut-off, three at one time point (18 months) and one at two time points ( $9 \& 18$ months). No TD infants scored below the cut-off to be of concern.

There was no linear or quadratic trend in the ASQ fine motor scores, $p=.37$ and .30 , respectively. However, group did contribute significantly to the prediction when included in the model, $b=-5.74(95 \% \mathrm{CI}=-10$ to -1.1$), t(16.21)=-2.62, p=.019$. Because TD infants were coded in all models as 0 and at-risk infants as 1 , the negative coefficient for group indicates lower fine motor scores on average for at-risk infants (see Figure 1). No infants scored below the cut-off to be of concern in this measure, however. 


\section{CSBS Caregiver Questionnaire}

The 24 months data for the CSBS sounds clusters were not analysed because all infants in at least one of the groups performed at ceiling on the task resulting in no variability in scores. Growth curve analysis of the sounds cluster raw scores, excluding the 24 months time point, showed a significant linear trend, $b=2.32(95 \% \mathrm{CI}=1.2$ to 3.5$), t(20.19)=4.20, p$ $<.001$, but no quadratic trend, $p=.37$. Group was a significant predictor in the model, $b=-$ $1.96(95 \% \mathrm{CI}=-3.8$ to -0.1$), t(13.24)=-2.22, p=.04$, however, there was no group by time interaction, $p=.411$. Therefore, the growth curve for speech sound development differs between at-risk and TD infants in terms of intercept (lower for at-risk infants) but not slope or rate of change with time (see Figure 2, which includes the 24 months data). Using a standard score cut-off of more than $1 S D$ below the mean, three at-risk participants showed cause for concern on the sounds cluster. Only one at-risk participant (P2) was below normal across all time points, the other two fell into the normal range by 15 months.

For the CSBS words cluster raw scores, the quadratic function over time was significant, $b=1.37(95 \% \mathrm{CI}=0.9$ to 1.9$), t(55.21)=5.58, p<.001$, not the linear function, $p$ $=.84$. While group was not a significant predictor, $p=.88$, the group by quadratic interaction was significant, $b=-.67(95 \% \mathrm{CI}=-1.3$ to -0.1$), t(12.89)=2.48, p=.028$. As shown in Figure 2 , the quadratic growth rate for at-risk infants was lower than the TD infants. On the words cluster two at-risk participants (P1 \& P2, see below) were below normal at two time points (both at 15 and 24 months) and one TD participant was below normal at one time point (15 months).

The CSBS object use raw scores revealed a significant linear trend, $b=6.39(95 \% \mathrm{CI}=$ 4.3 to 8.5$), t(18.72)=6.39, p<.001$, and no quadratic trend, $p=.28$. Neither group nor the interaction between group and the linear trend were significant predictors, $p=.57$ and $p=.34$, respectively. On the object use cluster six at-risk participants were below normal, five at only 
one time point and one at two time points. Most low scores were achieved at the 9 and/or 12 months assessments.

\section{REEL3}

The linear and quadratic functions were significant in the growth model of the REEL3 receptive raw scores, $b=11.39(95 \% \mathrm{CI}=8.3$ to 14.4$), t(27.24)=7.64, p<.001$, and, $b=-$ $1.26(95 \% \mathrm{CI}=-2.2$ to -.03$), t(21.868)=-2.71, p=.013$, respectively. The negative coefficient for the quadratic relationship shows the rate of change decreased for the later time collection points (see Figure 3). Neither group, $p=.14$, nor group by linear trend interaction, $p=.71$, were significant contributors to the model. The number of at-risk infants below normal on the REEL3 receptive abilities (SS < 85) ranged from 0 (at 15 months) to 2 ( 9 months). One TD infant was below normal at 12 months.

In the REEL3 expressive raw scores, a significant overall linear trend was found, $b=$ $9.24(95 \% \mathrm{CI}=6.0$ to 12.5$), t(38.07)=5.69, p<.001$, group was a significant predictor when added to the model, $b=-7.59(95 \% \mathrm{CI}=-10.7$ to -4.5$), t(36.74)=-4.91, p<.001$, but there was no interaction between group and the linear trend, $p=.52$. The negative coefficient for group shows the growth curve had a lower intercept for at-risk compared to TD infants, but there was no significant difference in slope (Figure 3). The number of at-risk infants below normal in REEL3 expressive abilities was six at 9 months, five at 12 and 18 months, and three at 15 months. Two TD infants were below normal in expressive abilities, one at 12 and one at 18 months.

\section{Language Outcomes at 24 Months}

Analysis of the CDI at 24 months showed a mean expressive vocabulary for the TD infants of 450 words (SD 85, range 367-548) with a mean percentile rank of 75 (SD 16, range 55-93). In contrast, mean expressive vocabulary for the at-risk group was 191 words (SD 156, range 17-428), significantly lower than the TD group, $t(11)=3.6, p=.01, d=2.06$. The mean 
percentile rank for the at-risk group was 28 (SD 24, range <5-61). The mean percentile of sentence complexity from the CDI was significantly lower for the at-risk (M 35, SD 31, range $<10-92$ ) compared to TD infants (M 75 SD 14, range 55-95), $t(11)=2.9, p=.02, d=1.66$.

Across all infants, the raw score for the CSBS sounds cluster at 9 months predicted CDI vocabulary scores at 24 months, $r(\mathrm{n}=13)=.68, p=.011$. The correlation with CDI sentence complexity was not statistically significant, $r(\mathrm{n}=13)=.54, p=.056$. Similarly, the sound cluster raw scores at 18 months predicted CDI vocabulary, $r(\mathrm{n}=13)=.70, p=.007$, but not sentence complexity, $r(\mathrm{n}=13)=.53, p=.06$.

\section{Single-Case Analyses of At Increased-Risk Infants}

At 24 months two at-risk infants (P1 \& P2), and no TD infants, had communication skills that were not developing appropriately for their age. Both P1 and P2 were less than the $5^{\text {th }}$ percentile in expressive vocabulary and $10^{\text {th }}$ percentile in sentence complexity, producing fewer than 50 single words (17 words for P1 and 32 for P2) and no two-word combinations. The same criteria have been used in previous studies to identify expressive language delay at 2 years (e.g., Rescorla \& Alley, 2001). These infants are, therefore, at increased-risk of ongoing speech and/or language problems.

Table 1 provides CAS-related features described by Davis and Velleman (2000) for the infant-toddler age range that could be assessed in the present study, as well as scores on those measures for P1 and P2 across all time points. P2 shows the presence of all features, suggesting a pattern of increased risk of CAS: below normal on the CSBS sounds cluster from infancy consistent with significant gaps in phonetic repertoire, developed use of gesture in their early development, signs of late fine motor development in infancy using the CSBS object use cluster, and a clear gap in early receptive and expressive communication skills (using the REEL3 and symbolic and speech composites). In contrast, P1 fell into the normal range for the sounds cluster at all time points, does not appear to be delayed in achievement 
of motor milestones at 9 months (although they were below normal on the object use cluster at 12 months), was below normal in gesture use for communication purposes at 9 months (normalising after that), and did not show the expected receptive-expressive gap with poor expressive skills at the earliest time point (9 months), but did at later time points.

All other CSBS scores not reported in Table 1 were in the normal range for P1 except for the words cluster at 15 months (SS 6) and 24 months (SS 6), and emotion and eye gaze (SS 6) at 9 months, a finding that is consistent with their poor REEL3 receptive ability score at that age. P2 was also below normal on the words cluster at 15 months (SS 6) and 24 months (SS 6), with normal performance on the remaining CSBS measures.

\section{Dissociation between conceptual/receptive development and speech motor areas.}

Applying the RSDT procedure (Crawford et al., 2003), a significant classical dissociation between the social composite of the CSBS caregiver questionnaire (SS 17) and the CSBS sounds cluster (SS 5) was observed for P2, $t(7)=4.31, p<.001$. In particular, there was no significant difference to the TD infants on the social composite, but a significant deficit on the sounds cluster, and a significantly larger discrepancy between the two types of score than the TD sample distribution. P2 also showed a significant (classical) dissociation between receptive and expressive ability scores (with poorer expressive ability) on the REEL3 relative to the TD infants, $t(7)=6.19, p<.001$. No dissociation was found for P1 between the social composite (SS 11) and sounds cluster (SS 9) scores, $t(7)=0.67, p=.52$, and between the REEL3 ability scores, $t(7)=0.27, p=.79$.

Examining other at-risk participants showed one child (P4) who at 9 months was also significantly below the TD infants on the sounds cluster (SS 4) but not different on the social cluster (SS 9) and had met RSDT criteria for a classical dissociation, $t(7)=2.84, p=.025$. A dissociation between receptive and expressive ability scores on the REEL was also apparent (SS $85 \& 75$, respectively), $t(7)=3.508, p=.01$, although the receptive score was borderline 
normal. This child normalised, however, on all components on the CSBS by 12 months onwards.

\section{Discussion}

Emerging speech and language skills were tracked from 9 to 24 months of age in two groups of infants: eight at-risk participants who were biological siblings of children with a clinical diagnosis of CAS and a comparison group of eight infants with no such family history. The findings of this study show an underlying weakness in early verbal communication development, particularly in expressive language and speech motor skills, in infants with a family history of CAS. Expressive, but not receptive, language was poorer in the at-risk group compared to the TD group. More than half at-risk infants showed below normal REEL3 expressive ability scores at most time points. Early prelinguistic expressive ability (e.g., at 9 months) typically encompasses broad conceptualisations of language, including vocalisation, babbling and gesture use. As development progresses, expressive language is defined more by word use and the development of syntax and would therefore be sensitive to deficits at higher levels of processing than speech motor development. At 24 months the CDI scores showed the at-risk group had overall weaker skills in these areas as well. The significant group by quadratic interaction in the CSBS words cluster SS suggests an emerging or widening difference in expressive vocabulary between the TD and at-risk participants with age (cf. Koster et al., 2005). In contrast, the measure of speech sound development (CSBS sounds cluster) showed weaker skills across all time points. This difference in developmental trajectory between speech sound and expressive language skills could reflect an emerging constraint of early and ongoing weak speech production skills on the emergence of expressive language. The importance of early speech sound development 
for later language emergence is also suggested by finding the CSBS sounds cluster raw scores at 9 and 18 months predict productive vocabulary size at 2 years.

The observation of generally lower but variable scores on speech and language measures for the at-risk group is consistent with a verbal trait deficit proposed by Lewis and colleagues (2004). In their study of familial aggregation of speech and language disorders in the family members of children with CAS, siblings presented with a range of disorders including mild articulation problems, severe language and speech sound disorders, and CAS. The authors proposed that traits underlying CAS may be polygenic (cf. Alcock, Passingham, Watkins, \& Vargha-Khadem, 2000) leading to variation in presentation of the broader phenotype (Lewis et al., 2006). The present results suggest degrees of affectedness in early development even among those showing normal language at 2 years of age. The risk genes associated with CAS may load more heavily on deficits in speech motor and expressive language development than other domains of language development.

The at-risk participants also demonstrated significantly poorer fine motor skills. This finding is consistent with the close relationship between fine motor and speech motor development proposed to exist in normal development, as well as descriptions of motor coordination difficulties in children with speech sound disorders (Bradford \& Dodd, 1996; Piek, 2006). It is possible that any broader phenotype of CAS may include relatively poor fine motor development (cf. Amorosa, von Benda, Dames, \& Schäfersküpper, 1986).

\section{Early Development in Infants at Increased-Risk of CAS}

Two at-risk participants (P1 and P2) who had not achieved normal milestones of language development at 2 years of age were regarded as being at increased risk of ongoing language and speech problems. P2 showed signs consistent with a high risk of developing

CAS (Davis \& Velleman, 2000) as demonstrated by (a) poor speech motor development using standardised assessments at all time points, (b) evidence of late motor milestones but 
developed use of gesture for communication purposes, and (c) a significant dissociation with poor speech sound development but age-appropriate conceptual and receptive development at 9 months. These findings support the proposition that speech motor development can be selectively impaired or deficient in some children at-risk of subsequent CAS (see, also, Highman et al., 2012). If P2 eventually receives a diagnosis of CAS, this would be consistent with the hypothesis that CAS can originate from a core deficit in infancy in speech motor control (Maassen, 2002, Terband et al., 2009).

P1 on the other hand showed (a) age appropriate speech motor development at all time points, (b) evidence of an early delay in gesture use, (c) no dissociation between speech sound development and conceptual and receptive ability scores at 9 months. We suggest P1 has the hallmarks of being overall delayed in language development, consistent with children who are late talkers at 2 years of age (Rescorla \& Alley, 2001), rather than primarily speech impaired. If a language impairment persists, it would suggest an underlying deficit that does not directly implicate the speech motor system, such as reduced processing capacity for phonological and lexical acquisition (cf. Bishop, 2006).

\section{Limitations}

Because of the small sample size, even if it were ascertained that P2 did meet criteria for CAS at a later age, it would not be possible to generalise the early profile we observed to the broader CAS population. We cannot conclude deviant speech motor development has caused higher order expressive language deficits. The presence of language deficits in children with CAS could reflect co-morbidity or a broader aetiology consistent with the verbal trait deficit.

One other at-risk infant showed a dissociation pattern at 9 months similar to P2 between speech motor development and conceptual/receptive language skills, and yet did not show below normal development at 2 years of age. It would be of interest in future research to 
investigate predictors of ongoing speech deficits in children with this early profile of development.

A further limitation of the study was the lack of information on the older siblings with suspected CAS. Relying on a diagnosis by qualified speech-language pathologists who work with children with CAS on their caseload has some face validity. However, in view of the possible heterogeneity in the heritable phenotype explored in the present study, the results involving the group comparisons on the early presentation of infants at risk of CAS should be seen as preliminary.

\section{Conclusions}

The present study is one of the first to document the developmental trajectory of speech and language in infants with a family history of the low prevalence disorder CAS. Group comparisons, showing generally poorer speech and expressive language skills in the atrisk participants, as well as the observation of ongoing delayed and/or disordered communication ability in two at-risk participants, provided support for a broad verbal trait deficit hypothesis of developmental speech motor disorders (Lewis et al., 2004). The results also confirm that it is possible for a child with increased risk of CAS to show, prelinguistically, a dissociation between modalities consistent with a core deficit in emerging speech motor control with normal receptive and conceptual abilities. That is, while sharing in a broader phenotype, this does not exclude the possibility of a specific and more severe vulnerability or deficit originating in the speech motor system in some infants. It remains to be seen to what extent this profile is a defining feature of children diagnosed with CAS, and what additional protective factors may buffer infants with this early profile from ongoing speech deficits. The present study highlights the utility of longitudinal paradigms coupled with single-case analyses in the study of CAS, using knowledge of familial risk to identify infants for investigation. 


\section{References}

Alcock, K. J., Passingham, R. E., Watkins, K., \& Vargha-Khadem, F. (2000). Oral dyspraxia in inherited speech and language impairment and acquired dysphasia. Brain and Language, 75, 17-33.

Amorosa, H., von Benda, U., Dames, M., \& Schäfersküpper, P. (1986). Deficits in fine motor coordination in children with unintelligible speech. European Archives of Psychiatry and Neurological Sciences, 236, 26-30.

Bishop, D. V. M. (1997). Cognitive neuropsychology and developmental disorders: Uncomfortable bedfellows. The Quarterly Journal of Experimental Psychology, 50(4), 899-923.

Bishop, D. V. M. (2006). What causes specific language impairment in children? Current Directions in Psychological Science, 15(5), 217-221. DOI: 10.1111/j.14678721.2006.00439.x

Bradford, A., \& Dodd, B. (1996). Do all speech-disordered children have motor deficits? Clinical Linguistics \& Phonetics, 10(2), 77-101.

Bricker, D., Squires, J., Mounts, L., Potter, L., Nickel, R., Twombly, E., et al. (1999). Ages and stages questionnaire: User's guide (2nd ed.). Baltimore: Paul H Brooks Publishing Company.

Bzoch, K. R., League, R., \& Brown, V. (2003). Receptive-expressive emergent language test (3rd ed.). Austin, Texas: PRO-ED.

Crawford, J. R., Garthwaite, P. H., \& Gray, C. D. (2003). Wanted: Fully operational definitions of dissociations in single-case studies. Cortex, 39, 357-370.

Davis, B. L., Jakielski, K. J., \& Marquardt, T. P. (1998). Developmental apraxia of speech: determiners of differential diagnosis. Clinical Linguistics \& Phonetics, 12(1), 25-45. 
Davis, B. L., \& Velleman, S. L. (2000). Differential diagnosis and treatment of developmental apraxia of speech in infants and toddlers. Infant-Toddler Intervention, 10(3), 177-192.

Evans, J. L. (2002). Variability in comprehension strategy use in children with SLI: A dynamical systems account. International Journal of Language and Communication Disorders, 37(2), 95-116. doi: 10.1080/1368282011011676 7

Fenson, L., Dale, P. S., Reznick, S., Thal, D., Bates, E., Hartung, J. P., et al. (1993). MacArthur Communicative Development Inventories. San Diego, CA: Singular Publishing Group.

Heck, R. H., Thomas, S. L., \& Tabata, L. N. (2010). Multilevel and longitudinal modelling with IBM SPSS. New York: Routledge.

Highman, C., Hennessey, N. W., Sherwood, M., \& Leitão, S. (2008). Retrospective parent report of early vocal behaviours in children with suspected childhood apraxia of apeech (sCAS). Child Language Teaching and Therapy, , 24(3), 285-306.

Highman, C. D., Leitão, S., Hennessey, N. W., Piek, J. P. (2012). Prelinguistic communication development in children with childhood apraxia of speech: A retrospective analysis. International Journal of Speech-Language Pathology, 14(1), $35-47$.

Hurford, D. P., \& Stutman, G. (2004). The 16th Mental Measurements Yearbook (Vol. 16). Lincoln: The Buros Institute of Mental Measurements.

Koster, C., Been, P. H., Krikhaar, E. M., Zwarts, F., Diepstra, H., \& Leeuwen, T. V. (2005). Differences at 17 months: productive language patterns in infants at familial risk for dyslexia and typically developing infants. Journal of Speech, Language, and Hearing Research, 48(2), 426- 438.

Levelt, W. J. M., Roelofs, A., \& Meyer, A. S. (1999). A theory of lexical access in speech production. Behavioral and Brain Sciences, 22, 1-75. 
Lewis, B. A., Freebairn, L. A., Hansen, A., Taylor, G. H., Iyengar, S., \& Shriberg, L. D. (2004). Family pedigrees of children with suspected childhood apraxia of speech. [Science Direct]. Journal of Communication Disorders, 37(2), 157-175.

Lewis, B. A., Freebairn, L. A., Hansen, A. J., Stein, C. M., Shriberg, L. D., Iyengar, S. K., Taylor, H. G. (2006). Dimensions of early speech sound disorders: A factor analytic study. Journal of Communication Disorders, 39, 139-157.

Maassen, B. (2002). Issues contrasting adult acquired versus developmental apraxia of speech. Seminars in Speech and Language, 23(4), 257-266.

Marion, M. J., Sussman, H. M., \& Marquardt, T. P. (1993). The perception and production of rhyme in normal and developmentally apraxic children. Journal of Communication Disorders, 26(3), 129-160.

Mitchell, P. R. (1995). A dynamic interactive developmental view of early speech and language production: application to clinical practice in motor speech disorders. Seminars in Speech and Language, 16(2), 100-109.

Oller, D. K., Eilers, R. E., Neal, A. R., \& Schwartz, H. K. (1999). Precursors to speech in infancy: The prediction of speech and language disorders. Journal of Communication Disorders, 32(4), 223-245.

Piek, J. P. (2006). Infant motor development. Champaigne, IL: Human Kinetics.

Rescorla, L., \& Alley, A. (2001). Validation of the language development survey (LDS): a parent report tool for identifying language delay in toddlers. Journal of Speech, Language and Hearing Research, 44(2), 434-445.

Shriberg, L. D., Aram, D., \& Kwiatkowski, J. (1997). Developmental apraxia of speech: Descriptive and theoretical perspectives. Journal of Speech, Language, and Hearing Research, 40(2), 273-285. 
Terband, H., Maassen, B., Guenther, F. H., \& Brumberg, J. (2009). Computational neural modelling of speech motor control in childhood apraxia of speech (CAS). Journal of Speech, Language, and Hearing Research, 52, 1595-1609. DOI: 10.10441092$4388(2009 / 07-0283)$

Wetherby, A. M., Allen, L., Cleary, J., Kublin, K., \& Golstein, H. (2002). Validity and reliability of the communication and symbolic behavior scales developmental profile with very young children. Journal of Speech, Language and Hearing Research, 45(6), $1202-1218$.

Wetherby, A. M., \& Prizant, B. M. (2002). Communication and Symbolic Behavior Scales Developmental Profile. Baltimore: Paul H Brookes Publishing Company.

\section{Acknowledgements}

We thank the speech-language pathologists and child-health nurses who assisted in recruiting families for this study, as well as the families involved in the study for their participation. We also thank two anonymous reviewers for their helpful feedback on an earlier version of this paper. 
Table 1

Presence of CAS-Related Characteristics in Infancy (Davis \& Velleman, 2000) for At-Risk

Participants $1(P 1)$ and $2(P 2)$

Feature

9

12

15

18

24 Present

Gaps in phonetic repertoire and lack of consonant-vowel babble

$\begin{array}{lllllll}\text { P1 CSBS Sounds } & 9 & 8 & 11 & 8 & 7 & \times \\ \text { P2 CSBS Sounds } & 5 & \mathbf{6} & \mathbf{5} & \mathbf{6} & \mathbf{6} & \checkmark\end{array}$

Developed use of gestures/signs

$\begin{array}{lcccccc}\text { P1 CSBS Gesture } & 6 & 12 & 11 & 11 & 17 & \mathbf{x} \\ \text { P2 CSBS Gesture } & 16 & 15 & 11 & 17 & 17 & \checkmark\end{array}$

Late motor milestones

$\begin{array}{lcccccc}\text { P1 CSBS Object Use } & 9 & \mathbf{6} & 8 & 10 & 9 & ? \\ \text { P2 CSBS Object Use } & \mathbf{3} & 11 & 11 & 12 & 10 & \checkmark\end{array}$

Receptive-expressive gap for vocal communication

$\begin{array}{lcccccc}\text { P1 REEL3 REC vs. EXP } & \mathbf{8 2 , 9 0} & 95, \mathbf{7 3} & n a & 103, \mathbf{7 9} & - & \times \\ \text { P1 CSBS Symb vs. Speech } & 11,8 & 10,8 & 10, \mathbf{6} & 12,8 & 10, \mathbf{6} & \mathbf{x} \\ \text { P2 REEL3 REC vs. EXP } & 95, \mathbf{6 5} & 90, \mathbf{7 0} & 97, \mathbf{6 0} & 105, \mathbf{7 5} & - & \checkmark \\ \text { P2 CSBS Symb vs. Speech } & 9, \mathbf{5} & 12, \mathbf{6} & 11, \mathbf{6} & 13,8 & 12, \mathbf{6} & \checkmark\end{array}$

Note. Scores in bold are below normal using >1 SD below the mean. CSBS Sounds, Gesture and Object Use are scale scores (M $10 S D$ 3) from the sounds, gesture, and object use clusters of the Caregiver Questionnaire of the Communication and Symbolic Behaviour Scales Developmental Profile (Wetherby \& Prizant, 2002). CSBS Symbolic and Speech are 
composite scale scores ( $M 10 S D$ 3). REEL3 REC and EXP are receptive and expressive ability scores from the Receptive-Expressive Emergent Language Test, $3^{\text {rd }}$ edition (Bzoch, et al., 2003). REEL3 was not administered at 24 months of age to the participants. $n a=$ not available, REEL3 scores could not be obtained at this age. 


\section{Figure Captions}

Figure 1. Mean Ages and Stages Questionnaire Communication (top panel) and Fine Motor (bottom panel) subtests for at-risk $(n=8)$ and typically developing $(n=8)$ infants from 9 to 18 months of age (with $95 \% \mathrm{CI}$ ).

Figure 2. Mean of raw scores from 9 to 24 months of age (with 95\% CI) for the CSBS sounds (top panel) and CSBS words (bottom panel) cluster for at-risk $(n=8)$ and typically developing $(n=8)$ infants.

Figure 3. Mean of raw scores from 9 to 24 months of age (with 95\% CI) for the REEL3 expressive (bottom panel) and receptive (top panel) ability subscales for at-risk $(n=8)$ and typically developing $(n=8)$ infants. 


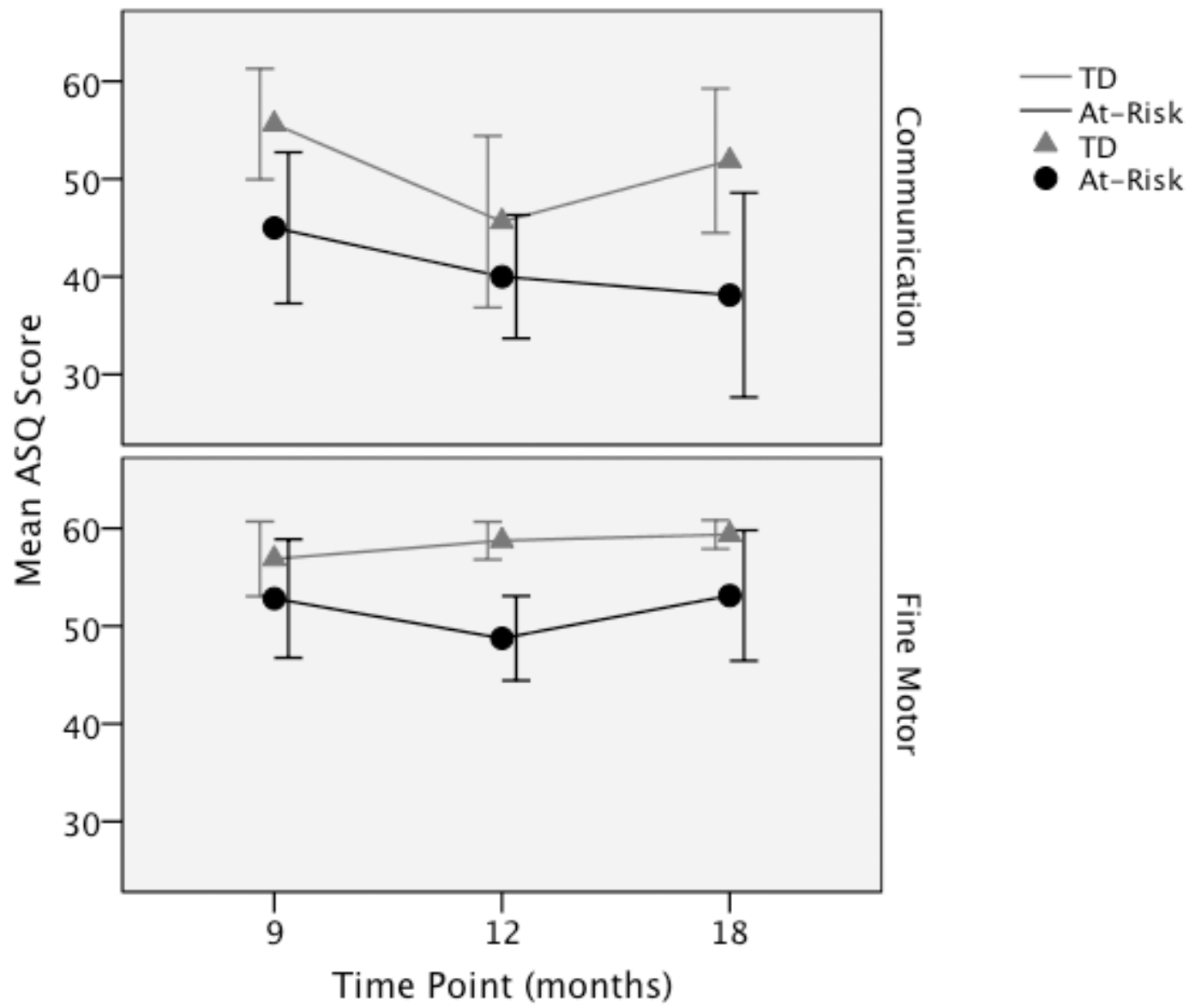




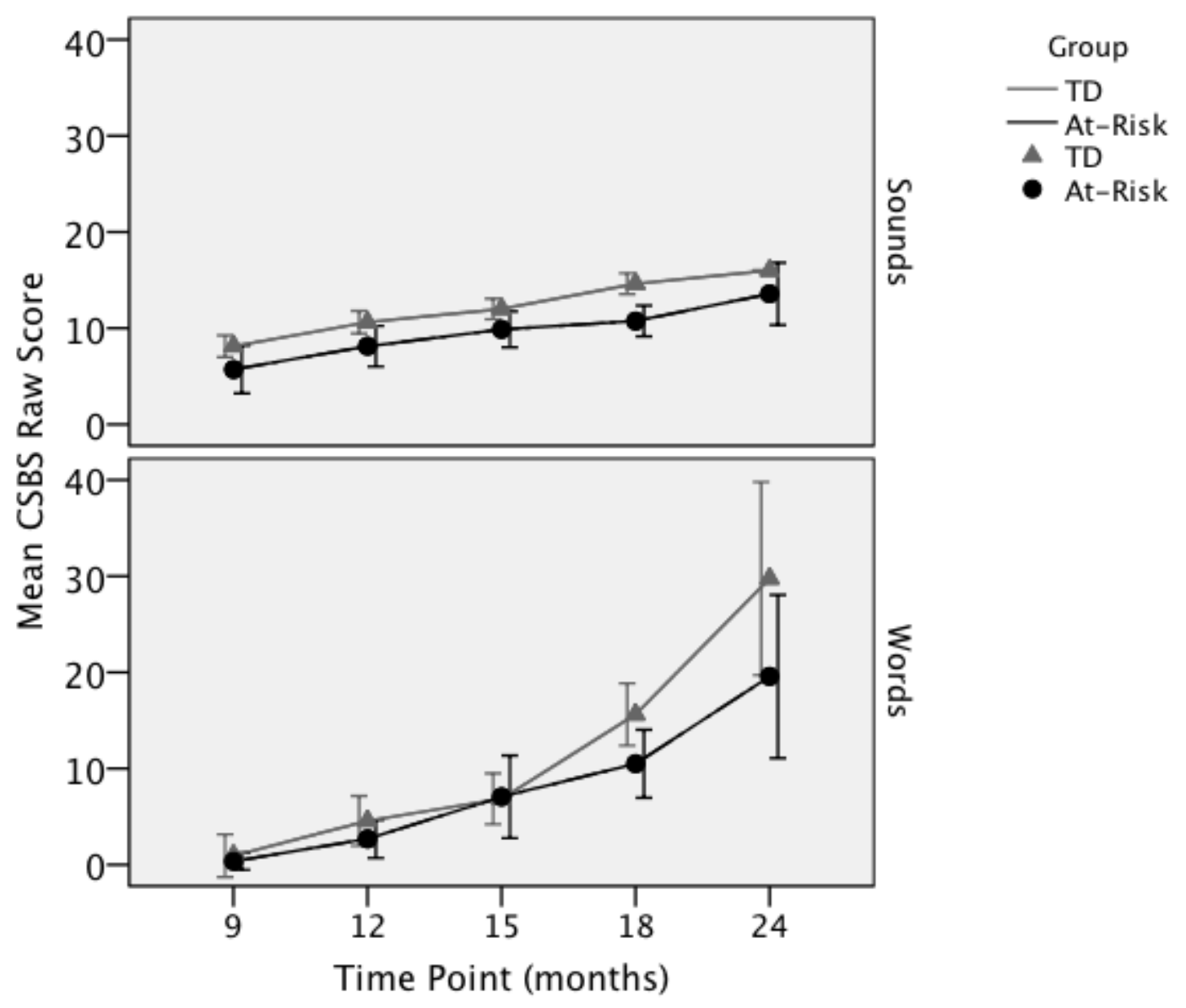




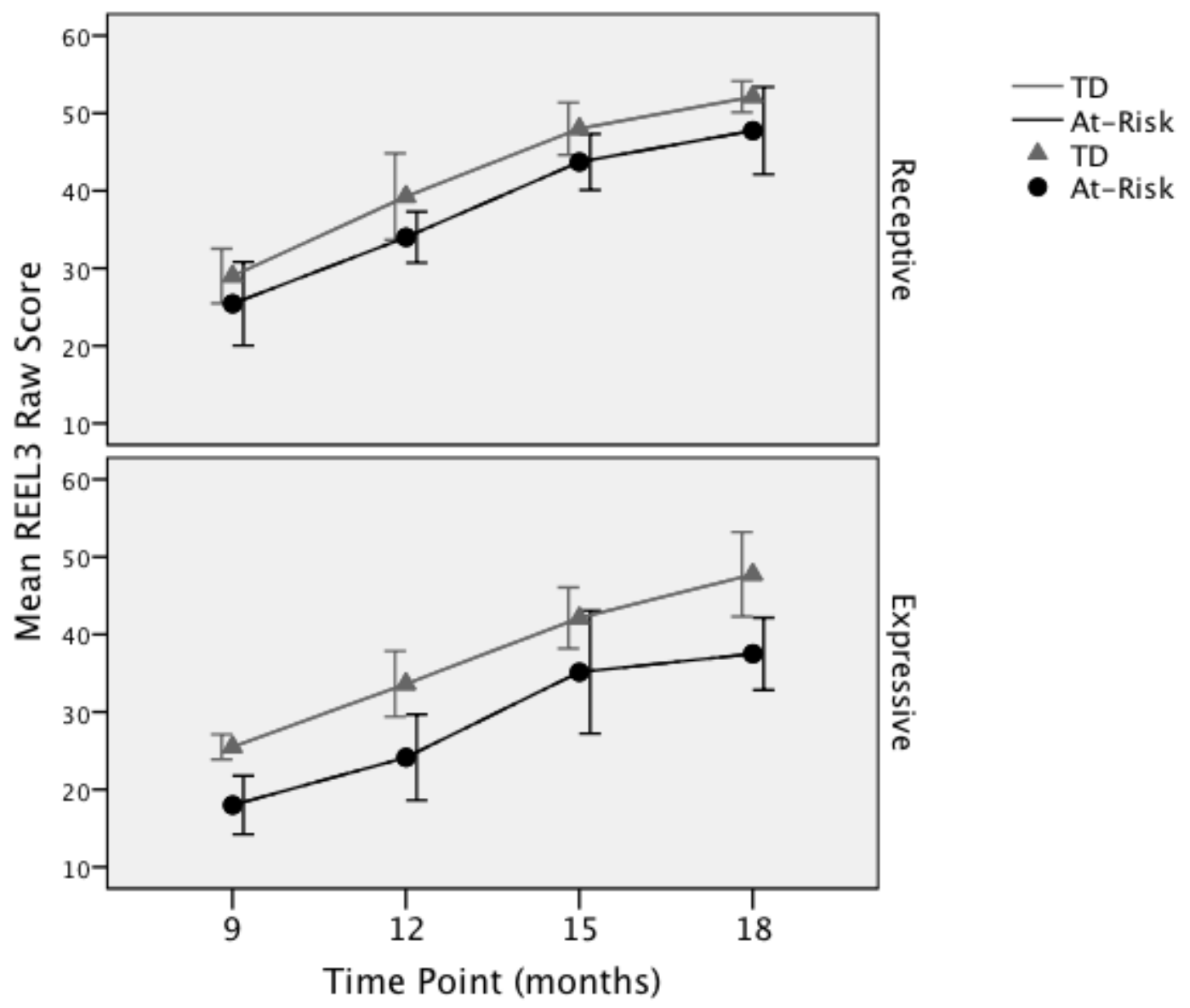

